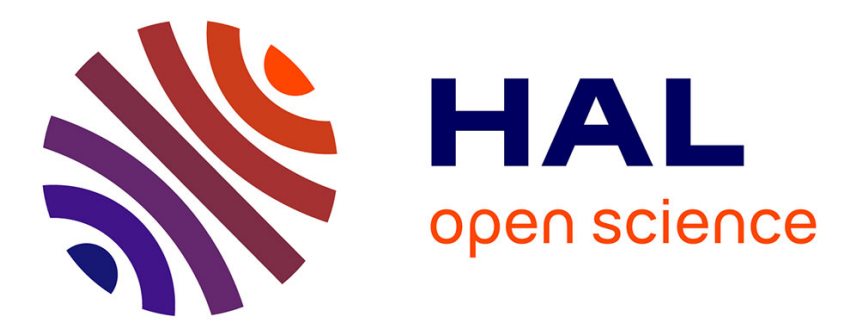

\title{
Theoretical study of stimulated Raman excitation of surface polaritons
}

\author{
R. Reinisch, N. Paraire, M. Chapet-Rousseau, S. Laval
}

\section{To cite this version:}

R. Reinisch, N. Paraire, M. Chapet-Rousseau, S. Laval. Theoretical study of stimulated Raman excitation of surface polaritons. Journal de Physique, 1977, 38 (11), pp.1457-1464. 10.1051/jphys:0197700380110145700 . jpa-00208719

\section{HAL Id: jpa-00208719 https://hal.science/jpa-00208719}

Submitted on 1 Jan 1977

HAL is a multi-disciplinary open access archive for the deposit and dissemination of scientific research documents, whether they are published or not. The documents may come from teaching and research institutions in France or abroad, or from public or private research centers.
L'archive ouverte pluridisciplinaire HAL, est destinée au dépôt et à la diffusion de documents scientifiques de niveau recherche, publiés ou non, émanant des établissements d'enseignement et de recherche français ou étrangers, des laboratoires publics ou privés. 


\title{
THEORETICAL STUDY OF STIMULATED RAMAN EXCITATION OF SURFACE POLARITONS
}

\author{
R. REINISCH, N. PARAIRE, M. CHAPET-ROUSSEAU and S. LAVAL \\ Institut d'Electronique Fondamentale (*), \\ Université Paris XI, Bâtiment 220, 91405 Orsay Cedex, France
}

(Reçu le 2 février 1977, révisé le 7 juillet 1977, accepté le 12 juillet 1977)

\begin{abstract}
Résumé. - On étudie l'excitation des polaritons de surface par effet Raman stimulé à l'aide de la méthode à deux faisceaux. La méthode employée permet de tenir compte facilement du fait que la polarisation non linéaire a une extension finie le long de l'interface et consiste à développer le champ E.M. à la fréquence des polaritons de surface sur la base formée par tous les modes propres de la structure considérée. En l'absence d'absorption, cette base est constituée d'un mode guidé, qui est le mode de polaritons de surface auquel nous nous intéressons ici, et d'une infinité de modes rayonnés. On détermine ainsi l'expression du champ E.M. à l'intérieur et à l'extérieur du domaine pompé. A la résonance, l'amplitude du mode de polaritons de surface à l'intérieur de la zone pompée croît linéairement en fonction de la coordonnée longitudinale. Les résultats concernant le mode de surface sont généralisés au cas où le milieu actif est absorbant. L'existence du phénomène de résonance permet de définir la courbe de dispersion des polaritons de surface excités par effet Raman dans un milieu absorbant.
\end{abstract}

\begin{abstract}
The excitation of surface polaritons by stimulated Raman scattering with the two beam method is studied theoretically. The method takes into account the finite spatial extent of the non-linear polarization along the interface. This method consists in expanding the E.M. field at the surface polariton frequency on a complete set of normal modes of the structure. When damping is neglected, this set consists of one surface polariton mode, and of an infinity of radiation modes. We determine the E.M. field inside and outside the pumped region. When resonance occurs, the amplitude of the surface polariton mode grows linearly with distance inside the pumped region. These results concerning the surface mode are subsequently generalized to absorbing active media. In that case the existence of the resonance phenomenon allows us to define the dispersion curve of the Ramanexcited surface polaritons.
\end{abstract}

1. Introduction. - Surface polaritons have been investigated by various experimental methods, which can roughly be classified into two main groups. In the first, a resonant coupling is observed between a surface excitation and an incident radiation field when they have almost equal frequencies and wave vectors; these linear processes include grating coupling [1] or attenuated total reflection [2]. In the second kind of experiment, non linear phenomena are used to obtain a resonant interaction, for example inelastic low energy electron diffraction [3], spontaneous [4] or stimulated [5] Raman scattering.

We are mainly concerned here with the excitation of surface polaritons by stimulated Raman scattering. We present a theoretical analysis of the non-

(*) Laboratoire associé au C.N.R.S. linear excitation of a surface polariton wave along a single interface using guided wave calculation techniques [6] : since we are interested in the amplification of surface waves propagating along the interface, it is convenient to expand the electromagnetic field into the normal modes of the unpumped guiding structure. Indeed, among these modes, which have a translational invariant amplitude and form an orthogonal complete set, is included the surface polariton mode. The non-linear excitation introduces a perturbation. We shall evaluate the variation of the mode amplitudes with distance, especially that of the surface wave.

We first outline the main features of this method, which is applicable to a wide range of guiding structures - including anisotropic (even non-reciprocal) and absorbing. media - and non-linear optical processes. 
We then discuss in detail the application of this method to the excitation of surface polaritons by a two-beam method (TBM) [7]. In that method the excitation beam (frequency $\omega_{\mathrm{L}}$ ) and the Stokes beam (frequency $\omega_{\mathrm{st}}$ ) are simultaneously sent in the nonlinear medium. Polaritons are excited at frequency $\omega=\omega_{\mathrm{L}}-\omega_{\mathrm{St}}$ [5]. We give the expression of the electromagnetic field at the polariton frequency, taking into account the simultaneous excitation of all the modes by the non-linear polarization. The surface polariton wave is predominantly excited when phase-matching occurs. Finally the influence of polariton damping is discussed.

2. General theory [6]. - Consider a four-vector $\phi$, the components of which are the transverse components of the electric and magnetic fields at frequency $\omega$.

$$
\boldsymbol{\phi}=\left(\mathbf{E}_{\mathrm{T}}, \mathbf{H}_{\mathrm{T}}\right) .
$$

In a guiding structure invariant by translation along the $z$ direction, normal modes of the form

$$
\phi_{l}=\left(\mathbf{E}_{\mathrm{T} l}, \mathbf{H}_{\mathrm{T} l}\right)=\boldsymbol{\varphi}_{l}(x, y) \mathrm{e}^{-j \beta_{l} z}
$$

can propagate.

$\beta_{l}$ is the longitudinal propagation constant for the $l$ th mode.

The set $\left\{\phi_{l}\right\}$ is assumed to be a complete one [6]. Thus any 4 -vector $\phi$, defined over the waveguide cross-section, can be expanded as follows :

$$
\left(\mathbf{E}_{\mathrm{T}}, \mathbf{H}_{\mathrm{T}}\right)=\sum_{l} C_{l}\left(\mathbf{E}_{\mathrm{T} l}, \mathbf{H}_{\mathrm{T} l}\right)
$$

where the summation includes both forward and backward travelling modes, and the symbol $\sum$ is used as a short hand notation. It applies to both guided and radiation modes $\left(=\sum\right.$ guided modes $+\int_{\text {The coefficients } C_{l} \text { are }}$ radiation modes).

The coefficients $C_{l}$ are determined using the following orthogonality relation, deduced from the Lorentz reciprocity relation integrated over a cross section plane [6].

$$
\left(\beta_{l}+\beta_{n}^{\mathrm{t}}\right)\left\langle\mathbf{u} .\left(\mathbf{E}_{l} \wedge \mathbf{H}_{n}^{\mathrm{t}}-\mathbf{E}_{n}^{\mathrm{t}} \wedge \mathbf{H}_{l}\right)\right\rangle=0
$$

$\mathbf{u}$ is a unit vector along the $z$-axis.

The brackets stand for an integral over the cross section :

$$
\langle\cdots\rangle=\iint \cdots \mathrm{d} x \mathrm{~d} y .
$$

The superscript $t$ denotes a mode corresponding to the adjoint structure, deduced from the original one by transposition of the dielectric permittivity $[\varepsilon]$ and magnetic permeability $[\mu]$ matrices.

Relation (2) can be rewritten under the following form :

$$
\left\langle\mathbf{u} \cdot\left(\mathbf{E}_{l} \wedge \mathbf{H}_{n}^{\mathrm{t}}-\mathbf{E}_{n}^{\mathrm{t}} \wedge \mathbf{H}_{l}\right)\right\rangle=N_{l} \delta_{n l}
$$

where $\delta_{n l}$ is the Kronecker symbol if at least one of the modes is a guided one and the Dirac $\delta$ function when both are radiation modes.

$\delta_{n l} \neq 0$ means that $n$ and $l$ are related by relation :

$$
\beta_{l}+\beta_{n}^{\mathrm{t}}=0 \text {. }
$$

From (3) we get :

$$
C_{l}=\frac{1}{N_{l}}\left\langle\mathbf{u} .\left(\mathbf{E}_{\mathrm{T}} \wedge \mathbf{H}_{n}^{\mathrm{t}}-\mathbf{E}_{n}^{\mathrm{t}} \wedge \mathbf{H}_{\mathrm{T}}\right)\right\rangle .
$$

When some perturbation is applied to the structure, the expansion (1) over the complete set $\left\{\phi_{l}\right\}$ remains valid [6], provided we use $z$-dependent coefficients :

$$
\left(\mathbf{E}_{\mathrm{T}}, \mathbf{H}_{\mathrm{T}}\right)=\sum_{l} C_{l}(z)\left(\mathbf{E}_{\mathrm{T} l}, \mathbf{H}_{\mathrm{T} l}\right) .
$$

Consider a perturbation due to a non-linear polarization $\mathfrak{P}^{\mathrm{NL}}$ at some given frequency $\omega$. The reciprocity relation must be generalized and its integration over a cross-section plane gives [6] :

$$
N_{l} \frac{\mathrm{d} C_{l}(z)}{\mathrm{d} z}=j \omega\left\langle\mathbf{E}_{n}^{\mathrm{t}} \cdot \mathscr{S}^{\mathrm{NL}}\right\rangle
$$

with the adjoint mode $n$ related to the mode $l$ by the relation (4). Eq. (7) shows that the non-linear polarization generally acts as a source for any mode of the structure.

The integration in (7) determines the expansion coefficients $C_{l}(z)$, and thereby, the transverse field components $\mathbf{E}_{\mathrm{T}}$ and $\mathbf{H}_{\mathrm{T}}$.

\section{Stimulated Raman excitation of surface polari-} tons. - Surface polaritons correspond to a guided wave which propagates along the interface between two media $[8,9]$. We will consider here phononpolaritons, although the derivation would be very similar for any other elementary excitation. The waves are guided by the interface, which coincides with the $(y, z)$-plane (Fig. 1). In the upper halfspace $(x>0)$, the dielectric permittivity $\varepsilon_{1}$ is assumed to be real, positive and independent of frequency. In the region $x<0$, we first assume a lossless nonlinear isotropic material, with real permittivity :

$$
\varepsilon_{2}(\omega)=\varepsilon_{\infty}+\sum_{j} \frac{S_{j} \omega_{j}^{2}}{\omega_{j}^{2}-\omega^{2}}
$$

$\varepsilon_{\infty}$ is the high frequency permittivity, $S_{j}$ and $\omega_{j}$ denote the oscillator strength and the transverse frequency of the $j$ th optical phonon mode respectively. The frequency ranges of interest for surface polaritons lie between the transverse and longitudinal phonon mode frequencies, where $\varepsilon_{2}(\omega)$ is negative. For simplicity, we assume that there is no dependence upon the $y$-coordinate, i.e. $\partial / \partial y=0$.

Let us first determine the 4-vectors $\phi_{l}$ corresponding to the normal modes for such a structure. 


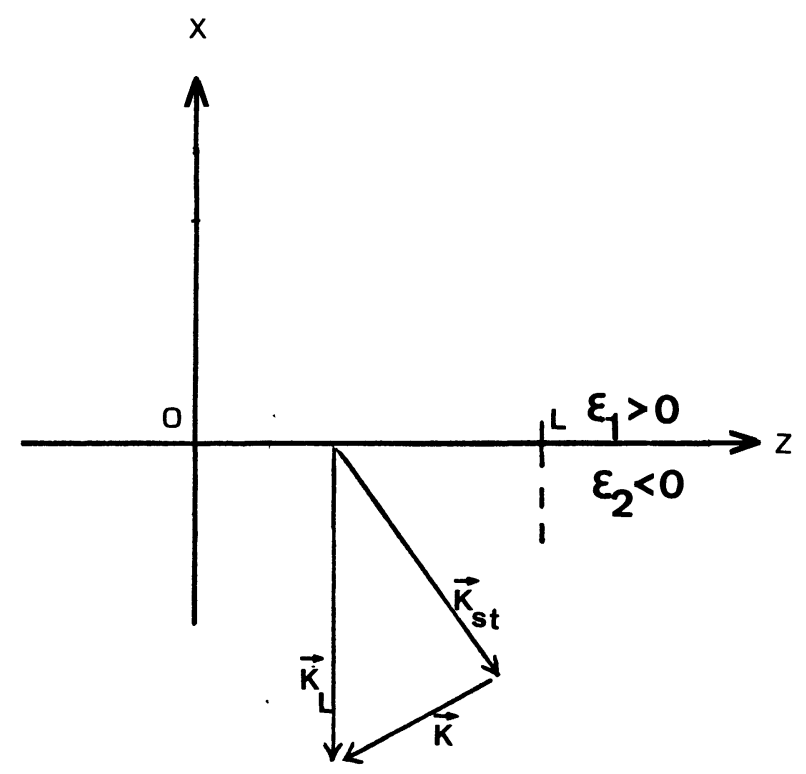

Fig. 1. - The geometry for surface polaritons.

In isotropic media, the normal modes are either TE or TM. Let $\phi_{l y}$ denote the electric field component $E_{l y}$, for a TE mode and the magnetic field $H_{l y}$ for a TM mode.

$\phi_{l y}(x, z)$ is a solution of the homogeneous propagation equation :

$$
\Delta \phi_{l y}+k_{q}^{2} \phi_{l y}=0
$$

where

$$
\begin{aligned}
& k_{q}^{2}=\frac{\omega^{2}}{c^{2}} \varepsilon_{q}, \quad q=1 \text { for } x>0 \\
& q=2 \text { for } x<0 .
\end{aligned}
$$

At the boundary $x=0$, the following conditions must be fulfiled :

$$
\begin{gathered}
\left.\phi_{l y}(x>0)\right|_{x=0^{+}}=\left.\phi_{l y}(x<0)\right|_{x=0^{-}} \\
\left.\sigma_{1} \frac{\partial \phi_{l y}(x>0)}{\partial x}\right|_{x=0^{+}}=\left.\sigma_{2} \frac{\partial \phi_{l y}(x<0)}{\partial x}\right|_{x=0^{-}}
\end{gathered}
$$

with $\sigma_{q}=1$ for TE modes

$\sigma_{q}=1 / \varepsilon_{q}$ for TM modes.

Forward and backward travelling modes will be derived independently in the same way, with a sign inversion of the longitudinal propagation constant.

The determination of the forward modes is given below :

For a forward mode, $\phi_{l y}(x, z)$ can be written :

$$
\phi_{l y}(x, z)=\left(A_{q} \mathrm{e}^{j \gamma_{l q} x}+B_{q} \mathrm{e}^{-j \gamma_{l q^{x}}}\right) \mathrm{e}^{-j \beta_{l z}}
$$

with

$$
\gamma_{l q}^{2}+\beta_{l}^{2}=k_{q}^{2}
$$

The index $l$ runs over both guided and radiation modes. Thus, $\gamma_{l q}^{2}$ and $\beta_{l}^{2}$ are real quantities (either positive or negative) since absorption is neglected.

1) Let us first consider the case $\beta_{l}^{2}>0$ : the corresponding modes propagate without any attenuation in the $z$-direction. Because surface polaritons exist only when $\varepsilon_{2}(\omega)$ is negative, we restrict the study to this situation.

$k_{2}^{2}<0$ prescribes $\gamma_{l 2}^{2}<0$, i.e. these modes always have exponentially decaying fields along the $x$-direction in the lower half-space. From eq. (12),

$$
\gamma_{l 1}^{2}=k_{1}^{2}-\beta_{l}^{2} .
$$

i) If $\beta_{l}<k_{1}, \gamma_{l 1}^{2}$ is positive and $0<\gamma_{l 1}<k_{1}$. The corresponding modes are radiated in the upper half space and can be expressed as :

$$
\begin{aligned}
& \begin{aligned}
\phi_{l y}(x>0, z) & = \\
& =A_{l}\left(\cos \gamma_{l 1} x+\frac{\alpha_{l 2} \sigma_{2}}{\gamma_{l 1} \sigma_{1}} \sin \gamma_{l 1} x\right) \mathrm{e}^{-j \beta_{l} z}
\end{aligned} \\
& \phi_{l y}(x<0, z)=A_{l} \mathrm{e}^{\alpha_{l 2} x} \mathrm{e}^{-j \beta_{l} z} .
\end{aligned}
$$

with $\alpha_{l 2}=j \gamma_{l 2}$.

ii) If $\beta_{l}>k_{1}, \gamma_{l 1}^{2}$ is negative and the mode has evanescent fields along the $x$-direction in both media : it is a guided mode. With $\alpha_{l 1}=j \gamma_{l 1}$, the boundary conditions (10) lead to :

$$
\frac{\alpha_{l 1}}{\alpha_{l 2}}=-\frac{\sigma_{2}}{\sigma_{1}} .
$$

This relation is inconsistent with eq. (12) for a TE mode $\left(\sigma_{1}=\sigma_{2}=1\right)$ : the guided mode has a pure TM character. Eq. (14a) can be written in the usual form of the dispersion relation for surface polaritons, which correspond to a single TM mode (index S) for a given frequency $\omega$ :

$$
\frac{\beta_{\mathrm{S}}^{2} c^{2}}{\omega^{2}}=\frac{\varepsilon_{1} \varepsilon_{2}}{\varepsilon_{1}+\varepsilon_{2}}
$$

The surface polariton field is given by :

$$
\begin{aligned}
& \phi_{\mathrm{S} y}(x>0, z)=A_{\mathbf{S}}^{\prime} \mathrm{e}^{-\alpha_{\mathbf{S} 1} x} \mathrm{e}^{-j \beta_{\mathbf{S}} z} \\
& \phi_{\mathbf{S} y}(x<0, z)=A_{\mathbf{S}}^{\prime} \mathrm{e}^{\alpha_{\mathbf{S} 2} x} \mathrm{e}^{-j \beta_{\mathbf{S}} z}
\end{aligned}
$$

2) Consider now the case $\beta_{l}^{2}<0$ : it corresponds to evanescent fields along the $z$-direction.

Let $\rho_{l}=+j \beta_{l}$.

From eq. (12),

$$
\gamma_{l 1}^{2}=k_{1}^{2}+\rho_{l}^{2},
$$

$\gamma_{l 1}^{2}$ is positive and then no guided modes exist along the interface for negative values of $\beta_{l}^{2}$.

$\gamma_{l 2}^{2}=-\left|k_{2}\right|^{2}+\rho_{l}^{2}$ is either positive or negative.

v) If $\rho_{l}<\left|k_{2}\right|, \gamma_{l 2}^{2}$ is negative and from eq. (12),

$$
k_{1}<\gamma_{l 1}<\sqrt{k_{1}^{2}+\left|k_{2}\right|^{2}} \text {. }
$$


The solution produces the same transverse variations as in eq. (13) :

$$
\begin{aligned}
& \phi_{l y}(x>0, z)= \\
& \quad=B_{l}\left(\cos \gamma_{l 1} x+\frac{\alpha_{l 2} \sigma_{2}}{\alpha_{l 1} \sigma_{1}} \sin \gamma_{l 1} x\right) \mathrm{e}^{-\rho_{l} z} \\
& \phi_{l y}(x<0, z)=B_{l} \mathrm{e}^{\alpha_{l 2} x} \mathrm{e}^{-\rho_{l} z} .
\end{aligned}
$$

vv) If $\rho_{l}>\left|k_{2}\right|, \gamma_{l 2}^{2}$ is positive and

$$
\gamma_{l 1}>\sqrt{k_{1}^{2}+\left|k_{2}\right|^{2}} \text {. }
$$

The corresponding modes are of the radiative type in both media, and appear as a superposition of orthogonal even (e) and odd (o) modes [10] with

$$
\begin{aligned}
& \phi_{l y}^{\mathrm{e}}(x>0, z)=B_{l \mathrm{e}}^{\prime} \cos \left(\gamma_{l 1} x\right) \mathrm{e}^{-\rho_{l z} z} \\
& \phi_{l y}^{\mathrm{e}}(x<0, z)=B_{l \mathrm{e}}^{\prime} \cos \left(\gamma_{l 2} x\right) \mathrm{e}^{-\rho_{l} z} \\
& \phi_{l y}^{\mathrm{o}}(x>0, z)=B_{l \mathrm{o}}^{\prime} \sin \left(\gamma_{l 1} x\right) \mathrm{e}^{-\rho_{l} z} \\
& \phi_{l y}^{\mathrm{o}}(x<0, z)=B_{l \mathrm{o}}^{\prime} \frac{\sigma_{1} \gamma_{l 1}}{\sigma_{2} \gamma_{l 2}} \sin \left(\gamma_{l 2} x\right) \mathrm{e}^{-\rho_{l} z} .
\end{aligned}
$$

These results are summarized in figure 2, where the field structure in each media is indicated as a function of $\gamma_{1}$ values. The only guided mode is the surface polariton, which corresponds to a particular value of the propagation constant $\beta_{\mathrm{S}}$, given by eq. (14b), and of $\gamma_{1}$. Besides this mode, which is pure TM, an infinity of TE and TM radiation modes exists, having either propagating or evanescent fields along $z$.

From the expressions of $\phi_{l y}$, the other components of the 4-vector $\phi_{l}$ for each normal mode can be determined. When a non-linear polarization exists in the non-linear medium 2, the expansion (6) is written :

$$
\begin{aligned}
\boldsymbol{\phi}(x, z)= & C_{\mathbf{S}}^{+}(z) \varphi_{\mathbf{S}}^{+}(x) \mathrm{e}^{-j \beta_{\mathbf{S}} z}+ \\
& +\int_{0}^{\infty} C^{+}\left(\gamma_{1}, z\right) \boldsymbol{\varphi}^{+}\left(\gamma_{1}, x\right) \mathrm{e}^{-j \beta z} \mathrm{~d} \gamma_{1} \\
& +C_{\mathbf{s}}^{-}(z) \varphi_{\mathbf{S}}^{-}(x) \mathrm{e}^{j \beta_{\mathbf{s}} z} \\
& +\int_{0}^{\infty} C^{-}\left(\gamma_{1}, z\right) \varphi^{-}\left(\gamma_{1}, x\right) \mathrm{e}^{j \beta z} \mathrm{~d} \gamma_{1}
\end{aligned}
$$

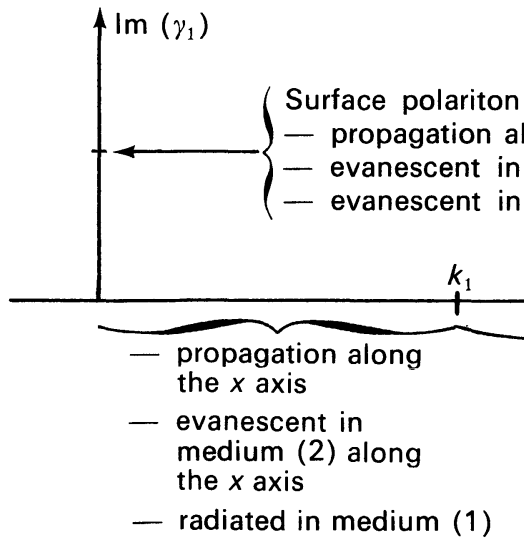

polariton mode :

- evanescent in medium (1) along the $x$ axis

the polariton frequency with the two incident fields, the amplitudes of which are varying according to the inhomogeneous propagation equations :

$$
\Delta \mathbf{E}_{\mathrm{L}, \mathrm{St}}+k_{\mathrm{L}, \mathrm{St}}^{2} \mathbf{E}_{\mathrm{L}, \mathrm{St}}=\frac{\omega_{\mathrm{L}, \mathrm{St}}^{2}}{c^{2}} \mathbf{P}^{\mathrm{NL}}\left(\omega_{\mathrm{L}, \mathrm{St}}\right)
$$

which would be solved together with eq. (7).

However, the non-linear coupling between eq. (7) and (21) can be removed by an approximation well adapted to the TBM [12], in which the two light beams must be symmetrically considered : as the interaction occurs, the higher frequency beam $\left(\omega_{\mathrm{L}}\right)$ is depleted while the lower frequency one $\left(\omega_{\mathrm{St}}\right)$ is amplified, and the product $E_{\mathrm{L}, i}(x, z) E_{\mathrm{St}, j}^{*}(x, z)$ can be assumed constant.

$\mathrm{Re}, \mathrm{Im}$ : respectively real and imaginary part.

FIG. 2. - Diagram giving the field characteristics for the different types of modes existing on the interface represented in figure 1. 
Let us write :

$$
\mathfrak{T}^{\mathrm{NL}}(\omega, x, z)=\mathbf{P}^{\mathrm{NL}}(\omega) \mathrm{e}^{-j \mathbf{K} . \mathbf{r}}
$$

with $\mathbf{K}=\mathbf{k}_{\mathbf{L}}-\mathbf{k}_{\mathbf{S t}}$.

Eq. (7) gives :

$$
\begin{aligned}
& N^{ \pm}\left(\gamma_{1}\right) \frac{\mathrm{d} C^{ \pm}\left(\gamma_{1}, z\right)}{\mathrm{d} z}= \\
& \quad=j \omega\left\langle\mathbf{E}^{ \pm \mathrm{t}}\left(\gamma_{1}^{\prime}, x\right) . \mathbf{P}^{\mathrm{NL}} \mathrm{e}^{-j \boldsymbol{K}_{x} x}>\mathrm{e}^{-j\left(\boldsymbol{K}_{z} \mp \beta\right) z}\right.
\end{aligned}
$$

(with $\beta^{t}\left(\gamma_{1}^{\prime}\right)+\beta\left(\gamma_{1}\right)=0$ ) and its integration leads to

$$
C^{ \pm}\left(\gamma_{1}, z\right)=\frac{u^{ \pm}\left(\gamma_{1}\right)}{K_{z} \mp \beta} \mathrm{e}^{-j\left(K_{z} \mp \beta\right) z}+D^{ \pm}\left(\gamma_{1}\right)
$$

where $D^{ \pm}\left(\gamma_{1}\right)$ is a constant and

$$
u^{ \pm}\left(\gamma_{1}\right)=-\frac{\omega}{N^{ \pm}\left(\gamma_{1}\right)}\left\langle\mathbf{E}^{ \pm t}\left(\gamma_{1}^{\prime}, x\right) \cdot \mathbf{P}^{\mathrm{NL}} \mathrm{e}^{-j K_{x} x}\right\rangle
$$

A similar expression is obtained for the surface polariton mode :

$$
C_{\mathrm{S}}^{ \pm}(z)=\frac{u_{\mathrm{S}}^{ \pm}}{K_{z} \bar{\mp} \beta_{\mathrm{S}}} \mathrm{e}^{-j\left(K_{\mathbf{z}} \mp \beta_{\mathbf{S}}\right) z}+D_{\mathrm{S}}^{ \pm}
$$

$u_{\mathrm{s}}^{+}$is calculated in the appendix.

Each term in eq. (18) has then the following form :

$$
\phi_{l}^{ \pm}(x, z)=\left(\frac{u^{ \pm}}{K_{z^{\prime}} \mp \beta} \mathrm{e}^{-j K_{z} z}+D^{ \pm} \mathrm{e}^{\mp j \beta z}\right) \boldsymbol{\varphi}_{l}^{ \pm}(x)
$$

and corresponds to the superposition of a driven wave with wavevector $K_{z}$ directed along the interface, and of a free wave, similar to the normal mode.

The constants $D^{ \pm}$are derived from the boundary conditions. Assuming that the non-linear polarization is applied from $z=0$ to $z=L$ along the interface and that no wave enters the pumped region, field continuity yields :

$$
\begin{aligned}
\phi^{<}(x, 0) & =\phi(x, 0) \\
\phi^{>}(x, L) & =\phi(x, L)
\end{aligned}
$$

$\boldsymbol{\phi}^{<}$and $\boldsymbol{\phi}^{>}$are respectively the 4-vectors corresponding to the transmitted fields in the regions $z<0$ and $z>L$, where no non-linear polarization exists :

$$
\begin{aligned}
\boldsymbol{\phi}^{\gtrless}(x, z)= & C_{\mathrm{S}}^{\gtrless} \boldsymbol{\varphi}_{\mathrm{S}}^{ \pm}(x) \mathrm{e}^{\mp j \beta_{\mathrm{S} z}}+ \\
& +\int_{0}^{\infty} C^{\gtrless}\left(\gamma_{1}\right) \boldsymbol{\varphi}^{ \pm}\left(\gamma_{1}, x\right) \mathrm{e}^{\mp j \beta z} \mathrm{~d} \gamma_{1} .
\end{aligned}
$$

The orthogonality relation (2) is used to obtain :

$$
\begin{aligned}
& D_{\mathrm{S}}^{+}=-\frac{u_{\mathrm{S}}^{+}}{K_{\mathrm{z}}-\beta_{\mathrm{S}}}, \quad D_{\mathrm{S}}^{-}=-\frac{u_{\mathrm{S}}^{-}}{K_{\mathrm{z}}+\beta_{\mathrm{S}}} \mathrm{e}^{-j\left(K_{\mathrm{z}}+\beta_{\mathrm{S}}\right) \mathrm{L}} \\
& C_{\mathrm{S}}^{>}=-\frac{u_{\mathrm{S}}^{+}}{K_{\mathrm{z}}-\beta_{\mathrm{S}}}\left(1-\mathrm{e}^{-j\left(K_{\mathrm{z}}-\beta_{\mathrm{S}}\right) L}\right) \\
& C_{\mathrm{S}}^{<}=\frac{u_{\mathrm{S}}^{-}}{K_{z}+\beta_{\mathrm{S}}}\left(1-\mathrm{e}^{-j\left(K_{\mathrm{z}}+\beta_{\mathrm{S}}\right) L}\right)
\end{aligned}
$$

and similar expressions for the radiation mode coefficients. The solution can be written :

$$
\begin{aligned}
& - \text { for } z<0 \\
& \boldsymbol{\phi}^{<}(x, z)=\frac{u_{\mathbf{S}}^{-}}{K_{z}+\beta_{\mathrm{S}}}\left(1-\mathrm{e}^{-j\left(K_{z}+\beta_{\mathbf{S}}\right) L}\right) \boldsymbol{\varphi}_{\mathbf{S}}^{-}(x) \mathrm{e}^{j \beta_{\mathbf{S}} z}+\int_{0}^{\infty} \frac{u^{-}\left(\gamma_{1}\right)}{K_{z}+\beta}\left(1-\mathrm{e}^{-j\left(K_{\mathbf{z}}+\beta\right) L}\right) \boldsymbol{\varphi}^{-}\left(\gamma_{1}, x\right) \mathrm{e}^{-j \beta_{z}} \mathrm{~d} \gamma_{1} \\
& - \text { for } 0<z<L
\end{aligned}
$$

$$
\begin{aligned}
& \boldsymbol{\phi}(x, z)=\frac{u_{\mathrm{S}}^{+}}{K_{z}-\beta_{\mathrm{S}}}\left(\mathrm{e}^{-j K_{z} z}-\mathrm{e}^{-j \beta \mathbf{S} z}\right) \varphi_{\mathrm{S}}^{+}(x)+\int_{0}^{\infty} \frac{u^{+}\left(\gamma_{1}\right)}{K_{z}-\beta}\left(\mathrm{e}^{-j K_{z} z}-\mathrm{e}^{-j \beta z}\right) \varphi^{+}\left(\gamma_{1}, x\right) \mathrm{d} \gamma_{1}+ \\
& \quad+\frac{u_{\mathrm{S}}^{-}}{K_{z}+\beta_{\mathrm{S}}}\left(\mathrm{e}^{-j\left(K_{z}+\beta_{\mathbf{S}}\right) z}-\mathrm{e}^{-j\left(K_{z}+\beta_{\mathbf{S}}\right) L}\right) \varphi_{\mathrm{S}}^{-}(x) \mathrm{e}^{j \beta_{\mathbf{S}} z}+\int_{0}^{\infty} \frac{u^{-}\left(\gamma_{1}\right)}{K_{z}+\beta}\left(\mathrm{e}^{-j\left(K_{z}+\beta\right) z}-\mathrm{e}^{-j\left(K_{z}+\beta\right) L}\right) \varphi^{-}\left(\gamma_{1}, x\right) \mathrm{e}^{j \beta z} \mathrm{~d} \gamma_{1} \\
& \quad-\text { for } z>L \\
& \phi^{>}(x, z)=-\frac{u_{\mathrm{S}}^{+}}{K_{z}-\beta_{\mathrm{S}}}\left(1-\mathrm{e}^{-j\left(K_{z}-\beta_{\mathbf{S}}\right) L}\right) \varphi_{\mathrm{S}}^{+}(x) \mathrm{e}^{-j \beta_{\mathbf{S}} z}-\int_{0}^{\infty} \frac{u^{+}\left(\gamma_{1}\right)}{K_{z}-\beta}\left(1-\mathrm{e}^{-j\left(K_{z}-\beta\right) L}\right) \varphi^{+}\left(\gamma_{1}, x\right) \mathrm{e}^{-j \beta z} \mathrm{~d} \gamma_{1} .
\end{aligned}
$$

We must note here that a nonlinear polarization which has not the transverse variation of the surface mode not only excites this mode, but also all the radiation modes. Thus, in general, the EM field at frequency $\omega$ is a mixture of all the normal modes supported by the interface.

In expressions (27-29) $K_{z}$ and $\omega$ appear as two parameters which can be varied independently. When $K_{z}=\beta_{\mathrm{S}}$, which is just the phase matching condition, the forward surface polariton mode is resonantly excited. Its amplitude then grows linearly with $z$ (as it propagates along the $z$ direction) inside the pumped region.

In order to estimate the relative importance of the 
surface mode, when resonantly excited, and of the radiation ones, let us calculate, at the end of the pumped region $(z=L)$ the Poynting vector flow, $W$, through a band orthogonal to the $z$ direction, of infinite extent along the $x$ direction and with a unit-length width $(1 \mathrm{~cm})$ along the $y$ direction. According to eq. (3), we get :

$$
\begin{aligned}
& W=\frac{1}{4} N_{\mathrm{S}}\left|u_{\mathrm{S}}^{+}\right|^{2} L^{2}+ \\
& +\frac{1}{4} \int_{0}^{k_{1}} N\left(\gamma_{1}\right)\left|u^{+}\left(\gamma_{1}\right)\right|^{2} \frac{\left|1-\mathrm{e}^{-j\left(\beta_{\mathrm{S}}-\beta\right) L}\right|^{2}}{\left(\beta_{\mathrm{S}}-\beta\right)^{2}} \mathrm{~d} \gamma_{1}
\end{aligned}
$$

with :

$$
N_{\mathrm{S}}\left|u_{\mathrm{S}}^{+}\right|^{2}=\left|P_{x}^{\mathrm{NL}}\right|^{2} \frac{\omega \beta_{\mathrm{S}} \alpha_{1} \varepsilon_{1}}{\varepsilon_{0}\left(\varepsilon_{2}^{2}-\varepsilon_{1}^{2}\right)} \frac{1}{\alpha_{2}^{2}\left(\beta_{\mathrm{S}}\right)+K_{x}^{2}}
$$

and from eq. $(3,13)$ :

$$
\begin{aligned}
& N\left(\gamma_{1}\right)\left|u^{+}\left(\gamma_{1}\right)\right|^{2}= \\
& \quad=\left|P_{x}^{\mathrm{NL}}\right|^{2} \frac{\omega \beta \varepsilon_{1}}{\pi \varepsilon_{0}\left(\varepsilon_{2}^{2}+\varepsilon_{1}^{2} \frac{\alpha_{2}^{2}\left(\gamma_{1}\right)}{\gamma_{1}^{2}}\right)} \frac{1}{\alpha_{2}^{2}\left(\gamma_{1}\right)+K_{x}^{2}}
\end{aligned}
$$

where, for simplicity, we have assumed that the $\omega_{\mathrm{L}}$ and $\omega_{\mathrm{St}}$ incident beams are linearly polarized in such a way that $\mathbf{P}^{\mathrm{NL}}$ is directed along the $x$ axis.

From eq. (30), $W$ appears as the sum of the power $W_{\mathrm{S}}$ carried by the surface mode and the power $W_{\mathbf{R}}$ carried by the radiation modes for $0<\gamma_{1}<k_{1}$.

We compute $W_{\mathrm{S}}$ and $W_{\mathrm{R}}$ for a GaP-air interface from the scattering diagram depicted on figure 1 . The two incident beams $\left(\omega_{\mathrm{L}}=14403 \mathrm{~cm}^{-1}, \omega_{\mathrm{St}}\right)$ carry a $10 \mathrm{MW} / \mathrm{cm}^{2}$ power density; in eq. (8) [13] $j=1, \omega_{1}=367.3 \mathrm{~cm}^{-1}, S_{1}=1.8, \varepsilon_{\infty}=9.09$ and in eq. (19)

$$
\chi_{h i j}=\chi_{x i j}=d_{\mathrm{E}}\left(1+\frac{c \omega_{1}^{2}}{\omega_{1}^{2}-\omega^{2}}\right)
$$

with [14] $d_{\mathrm{E}}=9 \times 10^{-22} \mathrm{MKSA}$ and $C=-0.53$.

The curves $W_{\mathrm{S}}$ and $W_{\mathrm{R}}$ versus $L$ are reported on figure 3 for two frequencies $\left(\omega=370 \mathrm{~cm}^{-1}\right.$ and $\left.\omega=380 \mathrm{~cm}^{-1}\right)$. They clearly show that the power carried by the surface mode becomes rapidly larger than the radiated power. When the surface polariton frequency approaches the transverse optical mode frequency $\omega_{1}, \beta_{\mathrm{S}}$ decreases towards $k_{1}$, and then also towards the wavevectors of the radiation modes which are, consequently, more efficiently excited. Thus, the period of their power oscillations, which arise from the fact that radiation modes are not excited at resonance, increases. For a pumped region length smaller than about $1 \mathrm{~mm}$, the radiation mode contribution can be important, in particular for frequencies close to $\omega_{1}$. If the pumped region is longer than a few $\mathrm{mm}$, the surface mode is predomi-

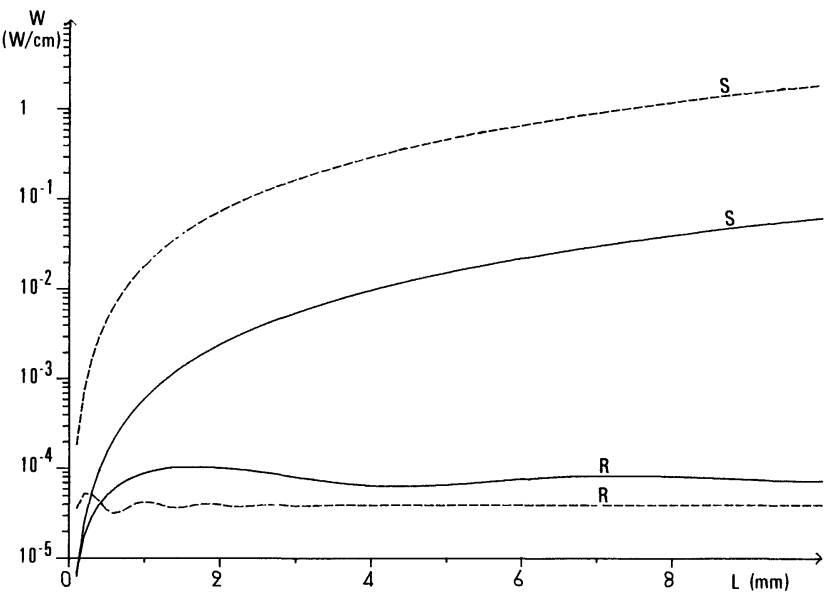

FIG. 3. - Power carried by the resonantly excited surface mode (S) and the radiation modes (R) versus the pumped region length, $L$, at two frequencies : $370 \mathrm{~cm}^{-1}$ : solid line $; 380 \mathrm{~cm}^{-1}$ : dashed line.

nant and the EM field at the surface polariton frequency can be approximated by the following expressions :

$$
\begin{aligned}
\phi^{<}(x, z) & \simeq 0 \\
\phi(x, z) & \simeq-j u_{S}^{+} \varphi_{S}^{+}(x) \mathrm{e}^{-j \beta_{S} z} z \\
\phi^{>}(x, z) & \simeq-j u_{S}^{+} \varphi_{S}^{+}(x) L \mathrm{e}^{-j \beta_{S} z} .
\end{aligned}
$$

The amplitude of the EM field keeps the same transverse dependence as the surface polariton mode. As we neglect damping, the transmitted field then keeps a constant amplitude.

Let us notice from the expression of $u_{\mathrm{s}}^{+}$that, because the polariton mode is TM, the $y$-component of the non-linear polarization does not contribute to its excitation.

4. Damping. - The surface polariton frequency lies in an IR absorption band of crystal, and the influence of damping must be considered. Let $\Gamma_{j}$ be the damping constant for the $j$ th optical mode. The dielectric permittivity $\varepsilon_{2}(\omega)$ is now a complex quantity :

$$
\varepsilon_{2}(\omega)=\varepsilon_{\infty}+\sum_{j} \frac{S_{j} \omega_{j}^{2}}{\omega_{j}^{2}-\omega^{2}+j \omega \Gamma_{j}} .
$$

Although the normal modes have been determined in a non-absorbing medium, a solution given by expressions (15) in which now $\beta_{\mathbf{S}}, \alpha_{\mathbf{S 1}}$ and $\alpha_{\mathbf{S} 2}$ are complex quantities exists and still represents the surface polariton mode with the following complex dispersion relation :

$$
\frac{\beta_{\mathrm{S}}^{2} c^{2}}{\omega^{2}}=\frac{\varepsilon_{1} \varepsilon_{2}}{\varepsilon_{1}+\varepsilon_{2}}
$$

where $\varepsilon_{2}$ is given by (34).

It is worth noting that, as $\alpha_{\mathbf{S 1 , 2}}$ take complex values, the surface mode propagation direction is not strictly 
parallel to the $z$-axis. Nevertheless this mode remains confined to the interface for small values of the damping constant.

The general theory in 2 is valid for absorbing media and can be used to get relations similar to (27-29). In the limit of weak absorption $\left(\Gamma_{j} \ll \omega_{j}\right)$ the resonance phenomenon described above will still take place. When it occurs, the main contribution to the EM field at frequency $\omega$ comes from the surface polariton mode. We then get :

$$
\begin{aligned}
\phi^{<}(x, z) & \simeq 0 \\
\phi(x, z) & \simeq \frac{u_{\mathrm{S}}^{+}}{K_{z}-\beta_{\mathrm{S}}}\left(\mathrm{e}^{-j K_{z} z}-\mathrm{e}^{-j \beta_{\mathbf{S}} z}\right) \varphi_{\mathrm{S}}^{+}(x) \\
\phi^{>}(x, z) & \simeq-\frac{u_{\mathrm{S}}^{+}}{K_{z}-\beta_{\mathrm{S}}}\left(1-\mathrm{e}^{-j\left(K_{\mathbf{z}}-\beta_{\mathbf{S}}\right) L}\right) \mathrm{e}^{-j \beta_{\mathbf{S} z}} \varphi_{\mathrm{S}}^{+}(x)
\end{aligned}
$$

$\beta_{\mathrm{S}}$ is complex but $K_{z}$, which is just the difference $k_{\mathrm{L} z}-k_{\mathrm{Stz}}$ between the $z$-components of the wavevectors at the optical frequencies (for which the medium 2 is transparent), is a real quantity. In the pumped region $\phi(x, z)$ is a sum of two waves : a free one whose amplitude decays exponentially as it propagates along the $z$ direction, and a driven one of constant amplitude. Whereas $\beta_{\mathrm{S}}$ obeys the dispersion relation (35), no dispersion relation is imposed between the driven wave vector $K_{z}$ and the frequency $\omega$, which can be varied independently. The amplitude $\left|\frac{u_{\mathrm{S}}^{+}}{K_{z}-\beta_{\mathrm{S}}}\right|$ of the driven wave being maximum for specific sets of values $\left(K_{z}, \omega\right)$, these values define the dispersion curve for surface polaritons when damping is taken into account. This definition is similar to those given for bulk polaritons [15] and is consistent with the analysis for surface polaritons studied by ATR [16] or by spontaneous Raman scattering [9].

5. Conclusion. - We have given a theory of nonlinear excitation of surface polaritons that takes into account the finite spatial extent of the pumped region along the interface. The theoretical method we have developed here is very convenient for this configuration. It shows that a non-linear polarization, which has not the transverse variation of one of the modes of the structure, generates, at the surface polariton frequency, an EM field which is a mixture of all the TE and TM modes supported by the interface. The surface polariton mode can be resonantly excited : this resonance phenomenon is used to define the dispersion curve of the TBM excited surface polariton in damped media.

Acknowledgments. - We are indebted to Professor J. A. Arnaud for several helpful discussions and a critical reading of the manuscript. It is a pleasure to thank Professor C. Vassalo for clarifying correspondence and discussions. We gratefully acknowledge the interest offered to this work by Professor C. Flytzanis and Professor J. C. Bolomey.

Appendix. - We calculate :

$$
u_{\mathrm{S}}^{+}=-\frac{\omega}{N_{\mathrm{S}}^{+}}\left\langle\mathbf{E}_{\mathrm{S}}^{+\mathrm{t}}(x) \cdot \mathbf{P}^{\mathrm{NL}} \cdot \mathrm{e}^{-j K_{x} x}\right\rangle .
$$

Media 1 and 2 are assumed to be non-absorbing. In that case [6] :

$$
\mathbf{E}^{\mathrm{t}}=-\mathbf{E}^{*}
$$

where $\mathbf{E}^{*}$ is the complex conjugate of $\mathbf{E}$

$$
u_{\mathrm{S}}^{+}=\frac{\omega}{N_{\mathrm{S}}^{+}}\left\langle\mathbf{E}_{\mathrm{S}}^{*}(x) \cdot \mathbf{P}^{\mathrm{NL}} \mathrm{e}^{-j K_{x} x}\right\rangle .
$$
TM :

We have seen that the surface polariton mode is

$$
\mathbf{E}_{\mathrm{S}}(x)=\left(E_{\mathrm{S} x}(x), 0, E_{\mathrm{Sz}}(x)\right) .
$$

From $\phi_{\mathrm{S} y}(x, z)$ given by eq. (15), we get :

$$
\begin{aligned}
& E_{\mathrm{S} x}=\frac{\beta_{\mathrm{S}}}{\omega \varepsilon_{q} \varepsilon_{0}} A_{\mathrm{S}}^{\prime} \mathrm{e}^{-\alpha_{q}|x|} \mathrm{e}^{-j \beta_{\mathbf{S} z}} \\
& E_{\mathrm{S} z}=-\frac{\alpha_{q}}{j \omega \varepsilon_{q} \varepsilon_{0}} \frac{x}{|x|} A_{\mathrm{S}}^{\prime} \mathrm{e}^{-\alpha_{q}|x|} \mathrm{e}^{-j \beta_{\mathbf{S} z}} .
\end{aligned}
$$

The surface mode amplitude is normalized such that : $N_{\mathrm{S}}^{+}=1$, where $N_{\mathrm{S}}^{+}$is given by the general formula (3). We get for the surface mode :

$$
N_{\mathrm{S}}^{+}=2 \mathcal{R e}\left\langle E_{\mathrm{S} x}(x, z) \cdot H_{\mathrm{S} y}^{*}(x, z)\right\rangle
$$

Re : real part.

Note that $N_{\mathrm{S}}^{+}$is four times the flow of the $z$-component of the Poynting vector. This property does not hold for absorbing media.

We have :

$$
N_{\mathrm{S}}^{+}=\left|A_{\mathrm{S}}^{\prime}\right|^{2} \frac{\omega}{\varepsilon_{0} \beta_{\mathrm{S}} c^{2} \alpha_{1}}\left(1-\frac{\varepsilon_{1}}{\varepsilon_{2}}\right) .
$$

Thus :

$$
\left|A_{\mathrm{S}}^{\prime}\right|=c \sqrt{\frac{\left|\beta_{\mathrm{S}}\right| \alpha_{1}}{\omega} \frac{\varepsilon_{0} \varepsilon_{2}}{\varepsilon_{2}-\varepsilon_{1}}}
$$

The phase of the coefficient $A_{\mathrm{S}}^{\prime}$ is chosen in such a way that $A_{S}^{\prime}$ is a real positive quantity : $A_{S}^{\prime}=\left|A_{S}^{\prime}\right|$.

We finally get the following expression for $u_{\mathrm{s}}^{+}$ (recall that $\mathbf{P}^{\mathrm{NL}}=0$ in medium 1 ) :

$$
u_{\mathrm{S}}^{+}=\frac{A_{\mathrm{S}}^{\prime}}{\varepsilon_{2} \varepsilon_{0}} \frac{\beta_{\mathrm{S}} P_{x}^{\mathrm{N}} \mathrm{L}-j \alpha_{2} P_{z}^{\mathrm{NL}}}{\alpha_{2}-j K_{x}}
$$

where $A_{\mathrm{S}}^{\prime}$ is given by (A.1). 


\section{References}

[1] Teng, Y. Y. and Stern, E., Phys. Rev. Lett. 19 (1967) 511 Richtie, R. H., Arakawa, E. T., Cowan, J. J. and Hamm, R. H., Phys. Rev. Lett. 21 (1968) 1530.

[2] OtTo, A., Z. Phys. 216 (1968) 398.

Marschall, N. and Fischer, B., Phys. Rev. Lett. 28 (1972) 811.

Barker, A. S., Phys. Rev. Lett. 28 (1972) 892 ; Phys. Rev. B 8 (1973) 5418.

LAgois, J. and Fischer, B., Phys. Rev. Lett. 36 (1976) 680.

[3] Iвасн, H., Phys. Rev. Lett. 24 (1970) 1416 ; Phys. Rev. Lett. 27 (1971) 253.

[4] Evans, D. J., Ushioda, S. and McMullen, J. D., Phys. Rev. Lett. 31 (1973) 369.

Prieur, J. Y. and Ushioda, S., Phys. Rev. Lett. 34 (1975) 1012.

[5] De Martini, F., Giulani, G., Mataloni, P., Palange, E. and SHEN, Y. R., Phys. Rev. Lett. 37 (1976) 440.

[6] Vassalo, C., Ecole d'Eté d'Electromagnétisme, Fascicule I : Généralités sur les ondes EM guides (CNET, Lannion) juillet 1973.

Arnaud, J. A., Beam and Fiber Optics (Academic Press, N.Y.) 1976.

MARCUSE, D., Light Transmission Optics (Van Nostrand N.Y.) 1972; Theory of Dielectric Optical waveguides (Academic Press, N.Y.) 1974.

SheVchenko, V. V., Continuous transitions in Open waveguides (The Golem Press) 1971.
Collin, R. E., Field theory of guided waves (McGraw-Hill, N.Y.) 1960 .

[7] Biraud-Laval, S., Reinisch, R., Paraire, N. and Laval, R., Phys. Rev. B, 13 (1976) 1797.

[8] Mills, D. L. and Burstein, E., Rep. Prog. Phys. 37 (1974) 817.

Nkoma, J., Loudon, R. and Tilley, D. R., J. Phys. C 7 (1974) 3547.

[9] Nkoma, J. and Loudon, R., J. Phys. C 8 (1975) 1950.

[10] MarCUSE, D., J.O.S.A. 64 (1974) 794.

[11] Bloembergen, N., Non-linear Optics (Benjamin Inc. N.Y.) 1965.

[12] Biraud-Laval, S., Thèse Série A No 1153, Université ParisSud, Centre d'Orsay, 1973.

[13] Marschall, N. and Fischer, B., Phys. Rev. Lett. 28 (1972) 811.

[14] Faust, W. L. and Henry, C. H., Phys. Rev. Lett. 25 (1966) 1265.

YARIV, A., Introduction to optical electronics, 2nd edition (Holt, Rinehart and Winston) 1976, p. 205.

[15] Benson, H. J. and Mills, D. L., Phys. Rev. B 1 (1970) 1678 Barker Jr., A. S. and Loudon, R., Rev. Mod. Phys. 44 (1972) 18.

Reinisch, R., Biraud-Laval, S. and Paraire, N., J. Physique 37 (1976) 227.

[16] BARKer Jr., A. S., Surf. Sci. 34 (1973) 62. 